\title{
Effects of an Impeller Rim and Radial Clearance on Energy Characteristics of an Axial Pump
}

\author{
D. G. Svoboda ${ }^{1}$, V. A. Golikov ${ }^{1}$, A. A. Zharkovskii ${ }^{1}$ and A. A. Klyuyev ${ }^{1}$ \\ ${ }^{1}$ Peter the Great St. Petersburg Polytechnic University, Polytechnicheskaya 29, St. Petersburg, \\ 195251, Russia
}

\begin{abstract}
The results of numerical and experimental research conducted in the Laboratory for Hydraulic Machinery Construction of Peter the Great St. Petersburg Polytechnic University are presented. The research is aimed at studying the effects of an impeller radial clearance and rim on the energy characteristic of low-pressure axial pumps of the specific speed $n s \approx 600$. It is shown that these design features of a flow duct have significant effects on stage parameters, and they have to be accounted for when verifying design and experimental characteristics of axial pumps.
\end{abstract}

\section{Introduction}

The flow energy in an axial pump is gained only on account of the force of the response from the lift acting on the fluid on the part of the blades upon rotation of the impeller and the transformation of the kinetic energy in a diffuser flow of the flow part [1]. Therefore, dynamic pumps of this type are unable to ensure high pressure gains, and the design features of the flow part can have significant effects on their energy and cavitation characteristics. A radial clearance between the pump impeller and casing wall is one of such design factors. An axial pump impeller can also have such element as a rim connecting the blades along the external contour. A rim enhances structural rigidity, increases natural oscillation frequency, prevents leakages of the fluid from the pressure side to the vacuum side of the blade. It is obvious that radial clearance, length of the rim, volume of leakage amounts, surface roughness can have effects on energy characteristics of an axial pump. The degree of these effects will be different for pumps with different specific speeds. This fact calls for research to determine optimal parameters of these design features, and also their accounting in verifying design and experimental characteristics of axial pumps. Certain works note also significant effects of radial clearance and rim on parameters of axial pumps with low specific speed $[2,3]$ and other machines $[4,5]$.

\section{Setting an objective and the goal of research}

The effects of these design parameters for axial pumps with specific speed $n_{\mathrm{s}} \approx 600$ have been investigated.

\footnotetext{
*Corresponding author: svoboda.dmitry@gmail.com
} 
Figure 1 shows solid models of vane systems of an axial pump on the basis of which in SolidWorks graphic editor 3D models have been constructed that imitate the volume of the wet end inside of which there is fluid flow being studied.

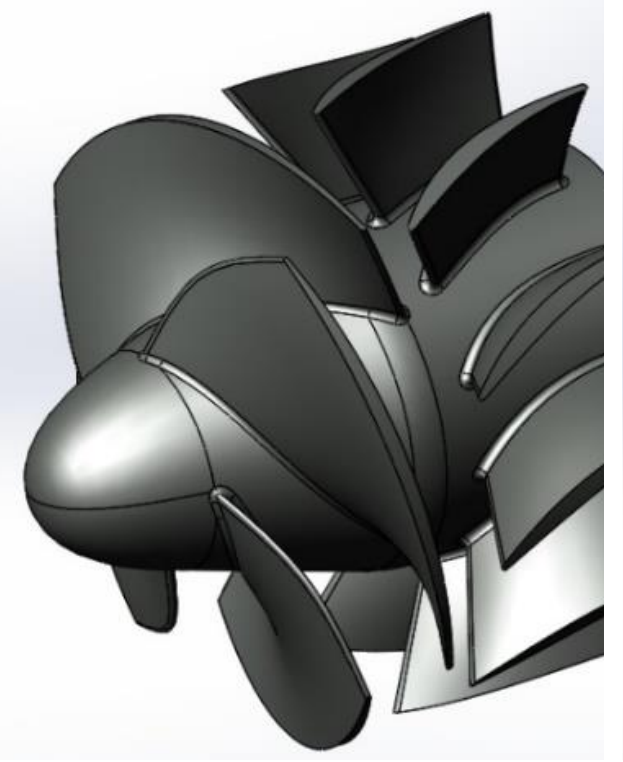

(a)

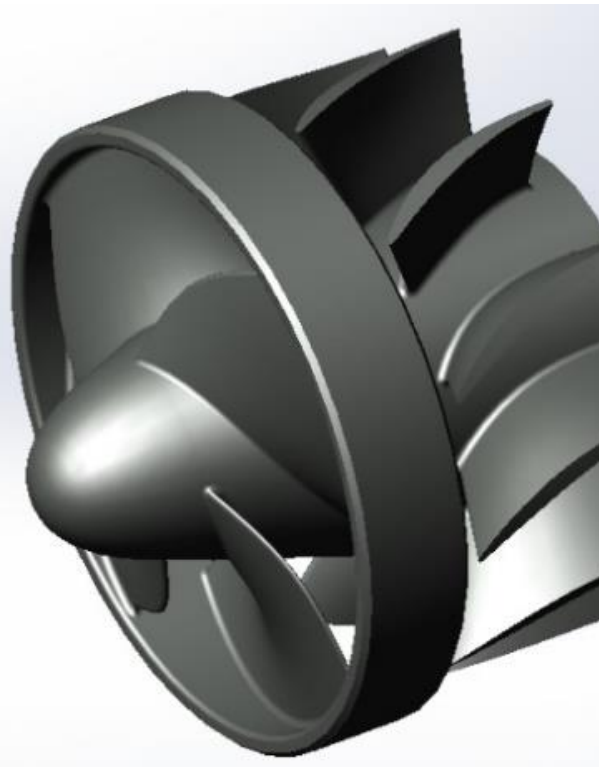

(b)

Fig. 1. Solid models of blade system of axial pump: without rim (a), with rim (b).

The computational region (figure 2) was modeled on the basis of the geometry of the flow part of a test bench placed in the Hydromechanical Engineering Laboratory of Peter the Great St. Petersburg Polytechnic University and included four regions - an inlet in a form of a confuser; an impeller domain; a guide vane domain combined with stator vanes and an outlet as a diffuser; a radial clearance domain.

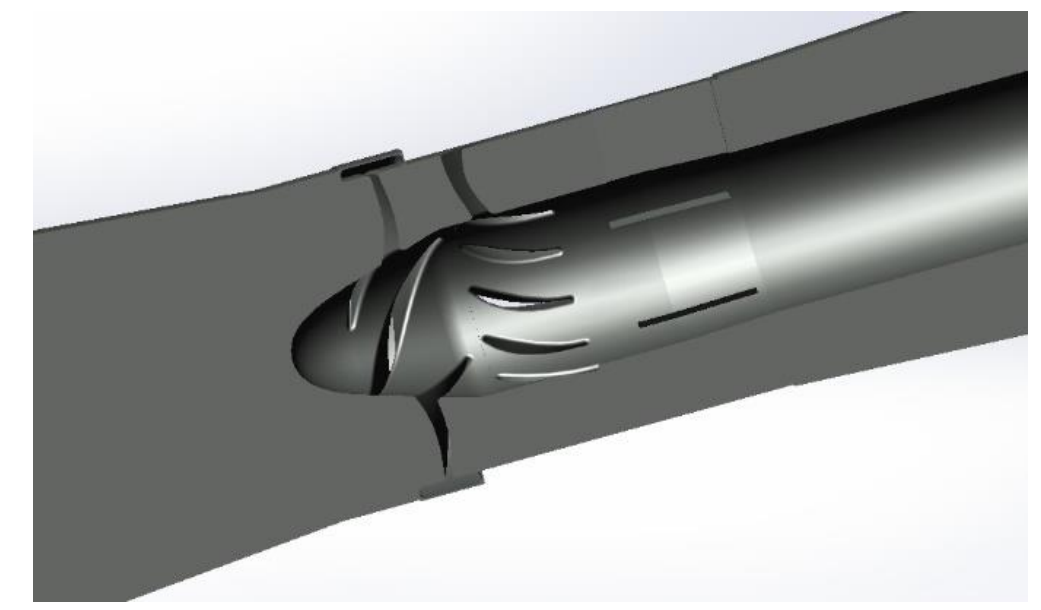

Fig. 2. Fluid flow model domain considering rim and radial clearance.

For an impeller with a rim the actual relative radial clearance determined on a bench along the impeller circumference using measuring probes was $\delta_{\text {rel }}=\delta / D_{\text {imp }}=0.0025-0.003$. For an impeller without a rim $\delta_{\text {rel }}=0.0025-0.005$. 


\section{Numerical research of energy characteristics of computational models}

Numerical research using 3D CFD methods $[6,7,8]$ was done in Ansys CFX software complex on Polytechnic - RSK Tornado heterogenetic cluster of the Computational Center of Peter the Great St. Petersburg Polytechnic University. The results of numerical simulation were verified on the base of the experimental data obtained on the test bench in the Hydromechanical Engineering Laboratory of Peter the Great St. Petersburg Polytechnic University.

To evaluate the effects of the impeller rim and the radial clearance numerical computations of several models were conducted:

- For an impeller with a rim. Relative radial clearances $\delta_{\text {rel }}=0.001$ and $\delta_{\text {rel }}=0.005$ were simulated. In the impeller region on the exterior flow boundary ("shroud" surface) a boundary condition "rotation" was assigned equal to the rotational frequency of the impeller.

- For an impeller without a rim. Relative radial clearances $\delta_{\text {rel }}=0.001$ and $\delta_{\text {rel }}=0.005$ were simulated. In the impeller region on the exterior flow boundary a boundary condition "counter-rotation" was assigned. Therefore, in the absolute coordinate system the rotational speed of the chamber wall was assumed to be zero.

Also, the following boundary conditions were assigned: at the inlet to the computational region - complete pressure 0 bars, at the outlet - mass flow rate corresponding to the operating conditions. The flow state was turbulent. A conventional two-equation $\mathrm{k}-\varepsilon$ turbulence model $[9,10]$ was used to close Reynolds-averaged equations.

According to the results of the numerical computations of viscous flow and experimental research under the working conditions of the pump $Q=(0.3-1) Q_{\text {nominal }}$ the energy characteristics of the stage were obtained presented in figures 3-6.

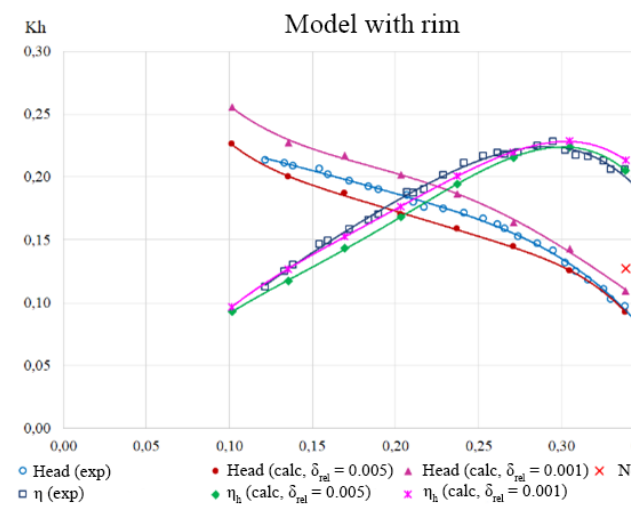

(a)

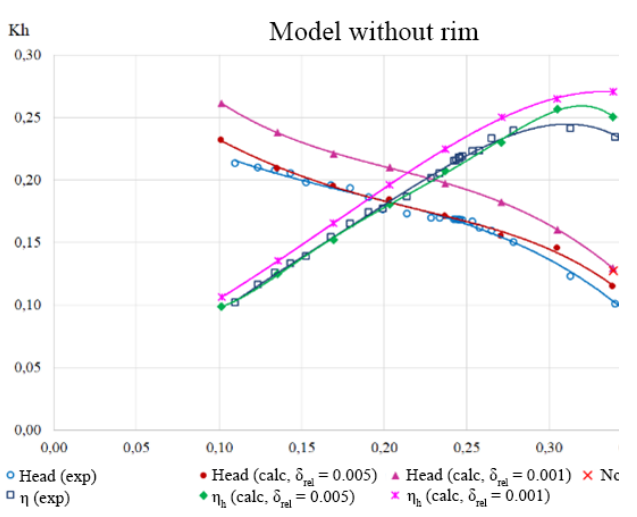

(b)

Fig. 3. Dimensionless energy characteristics: for model with rim (a) and model without rim (b). 


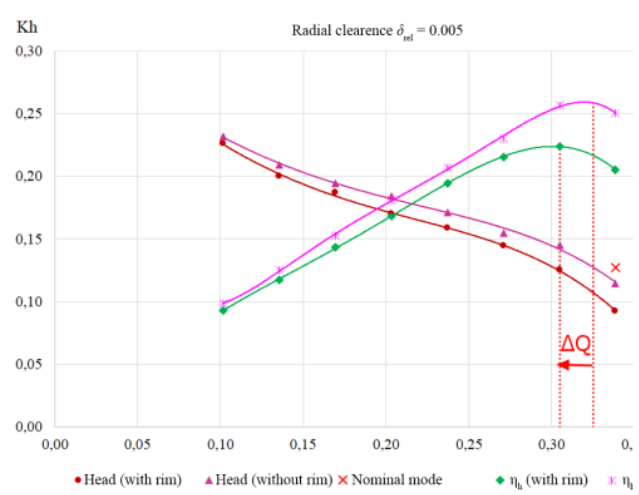

(a)

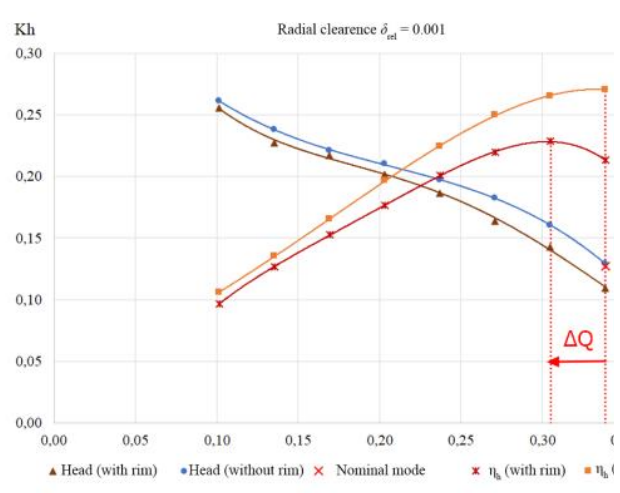

(b)

Fig. 4. Comparison of characteristics for models with rim and without rim: with radial clearance $\delta_{\text {rel }}=0.005$ (a) and with radial clearance $\delta_{\text {rel }}=0.001(\mathrm{~b})$.

The computed speeds and pressures in the flow being studied were visualized to analyze and evaluate the obtained results. Figures 5-6 presents profiles for a model with a rim and relative radial clearance $\delta_{\text {rel }}=0.005$ under the nominal operation conditions of a pump.

From figure 5 it follows that static pressure drop $\Delta p_{\text {st }}$ is formed in the clearance, the value of which is $\sim 5.2 \mathrm{~m}$.

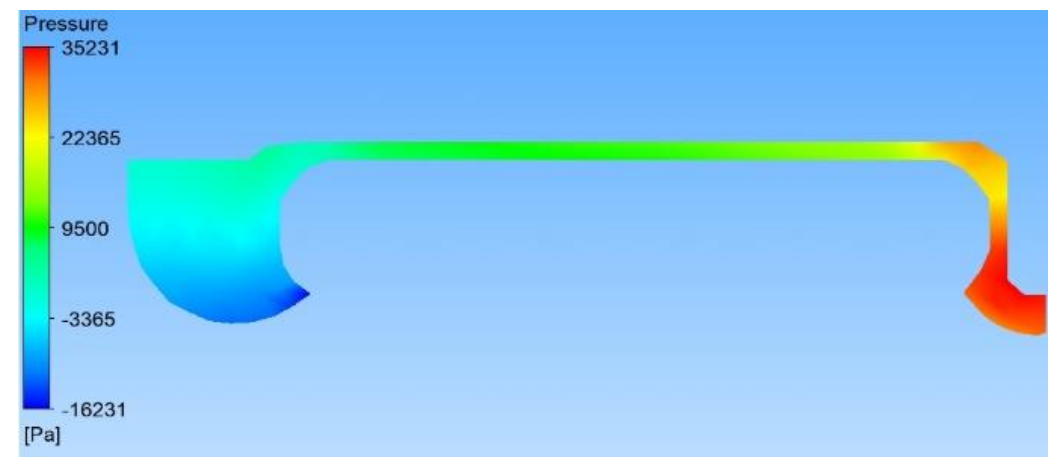

Fig. 5. Profile of static pressure in radial clearance.

As a result, a certain volume of fluids starts to leak through the radial clearance toward the inlet to the impeller. The leakage flow under the rated operation conditions of the pump is $\sim 2.3 \%$ of the entire feed, and the absolute speed reaches $W=12 \mathrm{~m} / \mathrm{s}$ (figure 6 ).

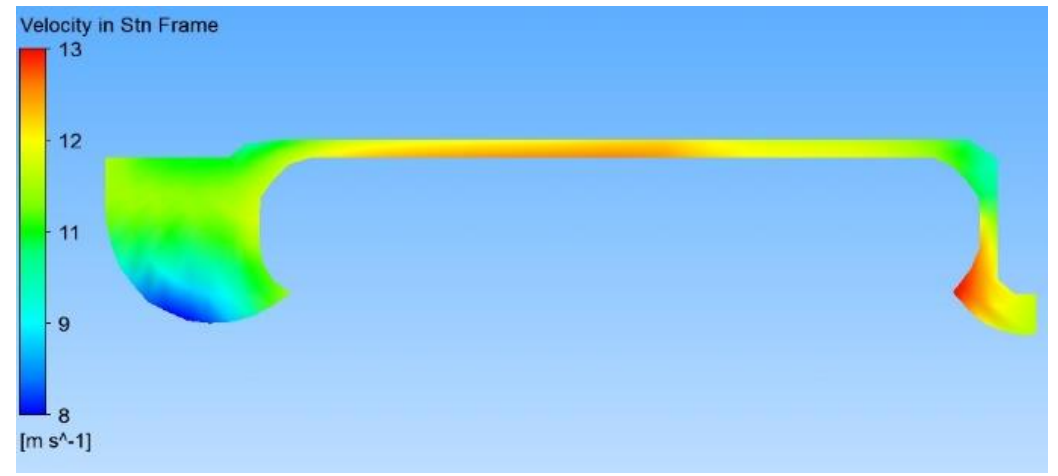

Fig. 6. Profile of absolute speed in radial clearance. 
Under part load conditions the static pressure drop increases, which leads to considerable increases in leakage flows. Under the conditions $Q=0.2 Q_{\text {nominal }}$ it is already $20 \%$ of the entire consumption that flows through the radial clearance (figure 7).

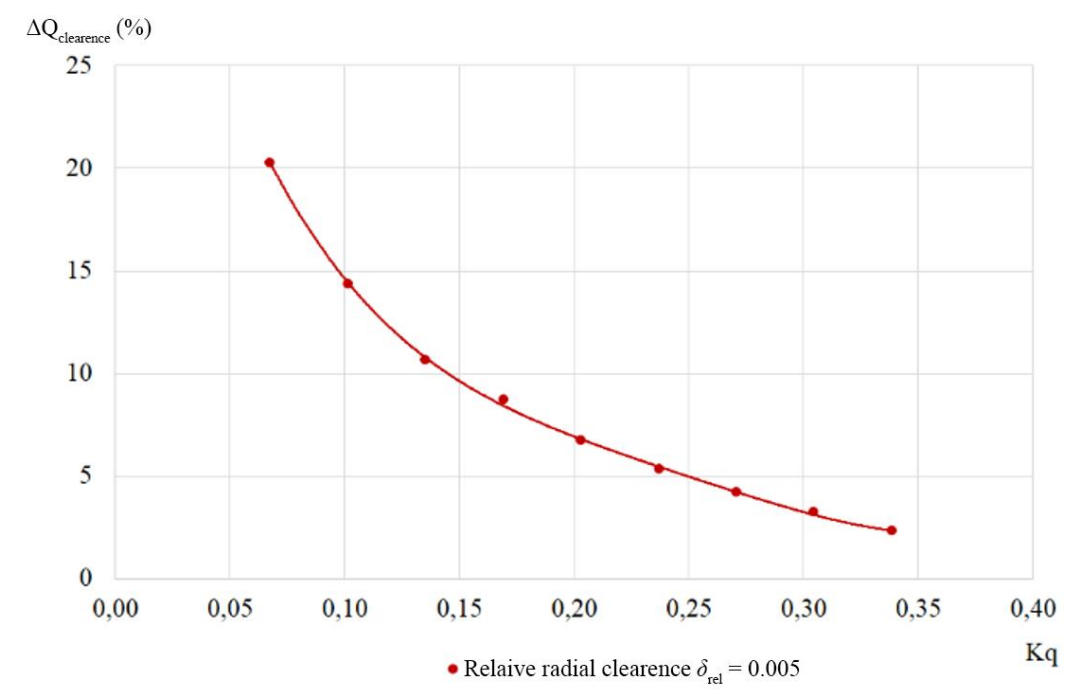

Fig. 7. Relative leakage flow

Thus, in the radial clearance there is constant circulation of the fluid volume $\Delta Q$ that does not participate in the energy gain and the creation of the pump power output and, in essence, increase volume losses. This leads to the fact that the optimum of the energy characteristics of the axial pump is shifted to the area of low flows, by the value of the volume of these leakages.

\section{Conclusions}

According to the results of the conducted research, the following conclusions can be drawn:

- Computational and experimental characteristics of the model of the axial pump being studied have a high degree of correlation, which confirms the appropriateness of the selected mathematical model.

- For a model without a rim an increase in relative radial clearance from 0.001 to 0.005 results in reduced efficiency of the pump under the rated conditions by $6 \%$.

- For a model with a rim an increase in relative radial clearance from 0.001 to 0.005 results in reduced efficiency of the pump under the rated conditions by $2.5 \%$.

- The presence of the impeller rim significantly increases losses of "disk" friction, which results in considerable reduction of the pump efficiency. Compared to a model without a rim the reduced pump efficiency under the rated conditions was $17 \%$ with relative radial clearance 0.001 . The efficiency was reduced by $13.5 \%$ with relative radial clearance 0.005 .

- For a model without a rim and with minimum clearance $\delta_{\text {rel }}=0.001$ the design optimal conditions coincide with rated conditions.

- For a model without a rim an increase in relative radial clearance from $\delta_{\text {rel }}=0.001$ to $\delta_{\text {rel }}=0.005$ results in the optimal conditions shifting toward less flow.

- For a model with a rim even with the minimum clearance $\delta_{\text {rel }}=0.001$ the optimum is shifted toward less flow. 
- The required pump pressure under the rated conditions is ensured only for a model without a rim and with minimum clearance $\delta_{\text {rel }}=0.001$.

- For axial pumps with specific speed $n_{\mathrm{s}} \approx 600$ it is recommended to maintain the relative clearance $\delta_{\text {rel }}=0.001$.

\section{References}

1. A. A. Lomakin, Centrifugal and axial pumps (Moscow: Mashinostroenie), 364 (1966)

2. A. S. Shapiro, Structure of real flow in centrifugal and axial pumps (Moscow: Moscow State Industrial University), 279 (2004)

3. D. G. Svoboda, Zharkovskii A. A. and Shchutskii S. Yu. Research of a rim and clearance influence on predictive characteristics of low-speed axial pump VIII Int. Scientific-tec. Conf. Hydraulic machines, hydropneumatic drives and hydropneumoautomatics, 2014

4. V. E. Shcherba, Shalai V. V., Grigor'ev A. V., Kondyurin A. Y., Lysenko E. A., Bazhenov A M and Tegzhanov A S 2019 Analysis of Results of Theoretical and Experimental Studies of the Influence of Radial Gaps in Stepped Slot Seal of Piston Hybrid Energy-Generating Machine Chemical and Petroleum Engineering 54 666-72

5. V. E. Shcherba, Shalai V. V., Kostyukov V. N., Naumenko A. P., Noskov A. S., Kondyurin A. Y. and Khait A. V. A Mathematical Model of the Working Processes of a Hybrid Power Displacement Piston Machine with Profiled Groove Seal Chemical and Petroleum Engineering 54 335-44, 2018

6. L. Shi, Zhang W., Jiao H., Tang F., Wang L., Sun D. and Shi W., Numerical simulation and experimental study on the comparison of the hydraulic characteristics of an axial-flow pump and a full tubular pump Renewable Energy 153 1455-64, 2020

7. H. Feng, Wan Y. and Fan Z. Numerical investigation of turbulent cavitating flow in an axial flow pump using a new transport-based model Journal of Mechanical Science and Technology 34 745-56, 2020

8. D. Zhang, Shi L., Shi W., Zhao R., Wang H. and van Esch B. P. M. Numerical analysis of unsteady tip leakage vortex cavitation cloud and unstable suction-side-perpendicular cavitating vortices in an axial flow pump International Journal of Multiphase Flow 77 244-59, 2015

9. K. J. Farrell and Billet M. L. A correlation of leakage vortex cavitation in axial-flow pumps Journal of Fluids Engineering, Transactions of the ASME 116 551-7, 1994

10. Muggli F. A., Holbein P. and Dupont P. CFD calculation of a mixed flow pump characteristic from shutoff to maximum flow Journal of Fluids Engineering, Transactions of the ASME 124 798-802, 2002 